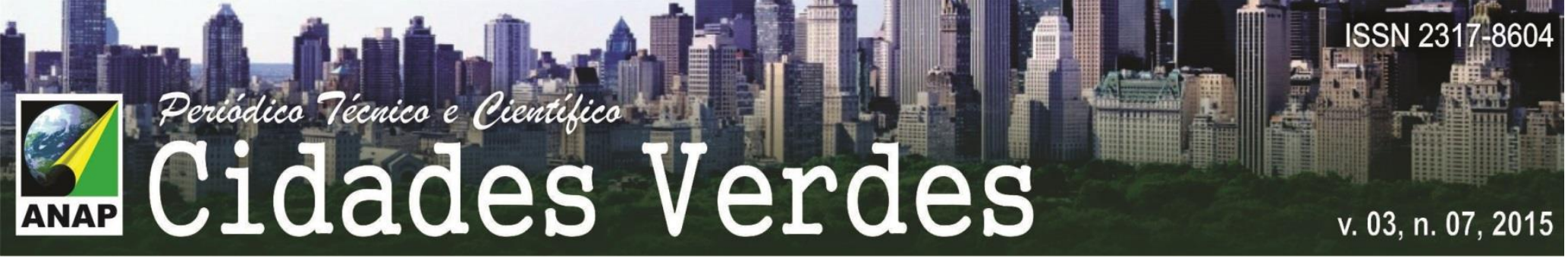

\title{
PROGRAMA CIDADES SUSTENTÁVEIS: O USO DE INDICADORES DE SUSTENTABILIDADE COMO CRITÉRIO DE AVALIAÇÃO DO AMBIENTE URBANO
}

\author{
Maria Eliza Alves Guerra ${ }^{1}$
}

Anaísa Filmiano Andrade Lopes ${ }^{2}$

\begin{abstract}
RESUMO
Entender as limitações e as potencialidades das cidades como meio de buscar a sustentabilidade urbana é um desafio que se apresenta na atualidade. O processo de urbanização causou problemas alarmantes como altos níveis de poluição, impactos associados ao transporte, ocupação desordenada, crescente quantidade de resíduos e uso indiscriminado dos recursos naturais, dentre outros. Desse modo, a presente pesquisa tem como objetivo propor a utilização de indicadores de sustentabilidade desenvolvidos pelo Programa Cidades Sustentáveis para o planejamento e gestão do ambiente urbano, inclusive para cidades pequenas, objeto de estudo de caso das autoras, referente ao mestrado da Faculdade de Arquitetura e Urbanismo da Universidade Federal de Uberlândia (FAUED - UFU). Esses indicadores podem servir de auxilio na instrumentalização das equipes técnicas das diversas secretarias das prefeituras ou de prestação de serviços públicos. A pesquisa se caracteriza como exploratória e descritiva, e o procedimento metodológico foi baseado na síntese crítica de conhecimentos disponíveis sobre o tema, mediante a análise e interpretação de bibliografia pertinente. Para tanto foram realizados levantamentos bibliográficos sobre as temáticas: sustentabilidade, cidades sustentáveis e indicadores de sustentabilidade. Os resultados apontam que distintos indicadores têm sido desenvolvidos para tentar medir o nível de sustentabilidade do ambiente urbano, no entanto, as múltiplas abordagens e conceitos de sustentabilidade, além da dificuldade de obtenção de informações e a complexidade dos cálculos tem dificultado a utilização adequada dessas ferramentas.
\end{abstract}

PALAVRAS-CHAVE: Ambiente Urbano. Sustentabilidade. Cidades Sustentáveis. Indicadores de Sustentabilidade

\section{SUSTAINABLE CITIES PROGRAM: The SUSTAINABILITY INDICATORS FOR USE AS ENVIRONMENTAL ASSESSMENT CRITERIA URBAN}

\footnotetext{
${ }^{1}$ Prof ${ }^{a}$ Dr ${ }^{\text {a }}$ da Faculdade de Arquitetura e Urbanismo, Universidade Federal de Uberlândia - UFU. mariaelizaguerra@faued.ufu.br

${ }^{2}$ Mestranda em Arquitetura e Urbanismo, Universidade Federal de Uberlândia - UFU. ana_isaandrade@hotmail.com
} 


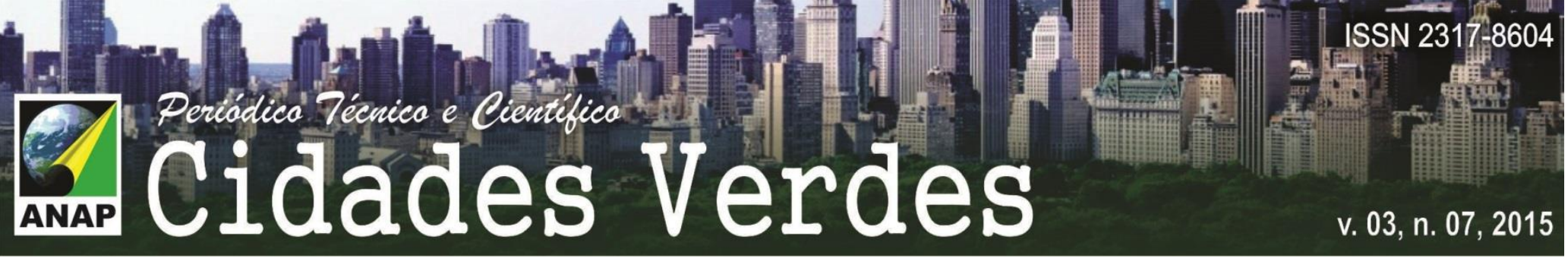

\begin{abstract}
Understand the limitations and potential of cities as a means of pursuing urban sustainability is a challenge before us today. The urbanization process caused alarming problems like high levels of pollution, impacts associated with transportation, disorderly occupation, increasing quantities of waste and indiscriminate use of natural resources, among others. Thus, this research aims to propose the use of sustainability indicators developed by the Sustainable Cities Programme for the planning and management of the urban environment, including small cities, case study object of the authors, referring to the Master of Architecture School and Urbanism at the Federal University of Uberlândia (FAUED - UFU). These indicators can serve as an aid in the instrumentalization of the technical teams of the various departments of municipalities or the provision of public services. The research is characterized as exploratory and descriptive, and the methodological approach was based on the critical synthesis of available knowledge on the subject, through the analysis and interpretation of relevant literature. Therefore, we conducted literature surveys on the themes: sustainability, sustainable cities and sustainability indicators. The results show that different indicators have been developed to try to measure the level of sustainability of the urban environment, however, multiple approaches and concepts of sustainability, besides the difficulty of obtaining information and the complexity of the calculations has hindered the proper use of these tools .
\end{abstract}

KEYWORDS: Urban Environment. Sustainability. Sustainable Cities. Sustainability Indicators

\title{
PROGRAMA CIUDADES SOSTENIBLES: LOS INDICADORES DE SOSTENIBILIDAD PARA EL USO COMO CRITERIOS DE EVALUACIÓN AMBIENTAL URBANA
}

\begin{abstract}
RESUMEN
Comprender las limitaciones y las potencialidades de las ciudades como un medio de alcanzar la sostenibilidad urbana es un desafío que se enfrenta hoy en día. El proceso de urbanización causo problemas alarmantes como los altos niveles de poluición, impactos asociados al transporte, la ocupación desordenada, la creciente cantidad de residuos, y el uso indiscriminado de los recursos naturales, entre otros. Por lo tanto, esta investigación tiene como objetivo proponer el uso de indicadores de sostenibilidad desarrolladas por el Programa de Ciudades Sostenibles para la planificación y gestión del medio ambiente urbano, incluidas las pequeñas ciudades, estudio de caso objeto de los autores, en referencia al Maestro de Escuela de Arquitectura y Urbanismo de la Universidad Federal de Uberlândia (FAUED - UFU). Estos indicadores pueden servir como una ayuda en la instrumentalización de los equipos técnicos de los distintos departamentos de los municipios o la prestación de servicios públicos. La investigación se caracteriza por ser exploratorio y descriptivo, y el enfoque metodológico se basa en la síntesis crítica de los conocimientos disponibles sobre el tema, a través del análisis y la interpretación de la literatura relevante. Por lo tanto, fueron realizados levantamientos bibliográficos de los temas: sostenibilidad, ciudad sostenibilidad y indicadores de sostenibilidad. Los resultados muestran que los diferentes indicadores se han desarrollado para ententar medir el grado de sostenibilidad del medio ambiente urbano, sin embargo, los múltiples enfoques y conceptos de sostenibilidad, además de la dificultad de obtención de informaciones y la complejidad de los cálculos ha obstaculizado el uso apropiado dessas herramientas.
\end{abstract}

PALAVRAS-CLAVE: Medio Ambiente Urbano. Sostenibilidad. Ciudad Sostenible. Indicadores de Sostenibilidad. 


\title{
1. INTRODUÇÃO
}

O processo de expansão urbana brasileira intensificou-se no século $X X$, principalmente a partir da Segunda Guerra Mundial. Essa expansão vem se apresentando ao longo do tempo de maneira desordenada especialmente nas grandes e médias cidades.

Côrrea (1997) imprime à cidade uma junção de alto nível cultural com a expressão do mais civilizado comportamento social. Mumford (1998) descreve a cidade como a mais avançada obra de arte da civilização humana. Para Rogers (2008), as cidades representam uma especialização das funções humanas, no entanto, as cidades trazem consigo todos os riscos reunidos, e de maneira intensa.

O modelo de desenvolvimento adotado na contemporaneidade, baseado no crescimento das relações de produção e consumo tem como implicações a degradação dos recursos naturais. Um problema crucial da atualidade é que o consumo desenfreado pode converter os recursos renováveis em não renováveis, ou então, renováveis somente depois de longo tempo (ROGERS, 2008). De acordo com esse mesmo autor, o fato é que a degradação ambiental se intensificou, as manchas da poluição, a industrialização, a expansão caótica de algumas cidades são evidencias de que a busca por riqueza está degradando o planeta.

Estamos enfrentando simultaneamente, a expansão populacional, a destruição dos recursos naturais, do meio ambiente e aumento nos níveis de desigualdade social e de concentração de riqueza.

\begin{abstract}
A urbanização tem-se caracterizado em especial no Brasil, como um processo não planejado, sobre 0 qual as ações da gestão são, normalmente, de caráter corretivo, objetivando situações problemáticas já estabelecidas, resultado das políticas urbanas locais, regionais e nacional até então adotadas. A dualidade entre o paradigma do crescimento e o da sustentabilidade tem transformado a administração destes espaços em tarefa das mais árduas (ROSSETO, 2003, p. 21).
\end{abstract}

Desta forma, o fortalecimento dos processos de gestão dos municípios é necessário para resgatar e manter a qualidade do ambiente urbano e de seus 
de prestação de serviços públicos, o presente trabalho propõe a utilização destes indicadores de sustentabilidade proposto pelo Programa Cidades Sustentáveis para o planejamento e gestão do ambiente urbano. O programa permite utilizar esses indicadores para avaliação em cidades pequenas ( com menos de $50 \mathrm{mil}$ habitantes), os mesmos serão utilizados na pesquisa das autoras, referente ao mestrado da Faculdade de Arquitetura e Urbanismo da Universidade Federal de Uberlândia (FAUED - UFU), como um estudo de caso para avaliar a sustentabilidade da cidade de Prata-MG, que possui uma população de aproximadamente 25.802 habitantes (IBGE, 2010).

Este programa é uma ferramenta importante pois permite traçar diagnósticos de diversas localidades, sejam elas de pequeno ou grande porte, utilizando-se os diversos indicadores (sociais, políticos, econômicos, culturais e ambientais).

\section{METODOLOGIA}

Esta pesquisa se caracteriza como sendo exploratória, pois segundo Gil (2002), as mesmas têm como objetivo proporcionar maior familiaridade com o problema, com vistas a torná-lo mais explícito. Na maioria dos casos envolvem: levantamento bibliográfico, entrevistas com pessoas que tiveram experiências práticas com o problema pesquisado e análise de exemplos que estimulem a compreensão.

Nesta pesquisa a coleta de dados e informações foi elaborada a partir da revisão bibliográfica de temáticas relacionadas ao ambiente urbano, sustentabilidade, cidade sustentável e indicadores de sustentabilidade. Para Minayo (2006), a pesquisa exploratória tem como objetivo principal o aprimoramento das ideias, dado que seu planejamento é bastante flexível e possibilita a consideração dos mais variados aspectos relativos ao fato estudado.

Esta pesquisa se caracteriza também como descritiva, tendo como foco central a descrição das características de determinadas populações ou fenômenos (GIL, 2002). No caso específico deste trabalho, acontecerá pela descrição dos indicadores de sustentabilidade. 


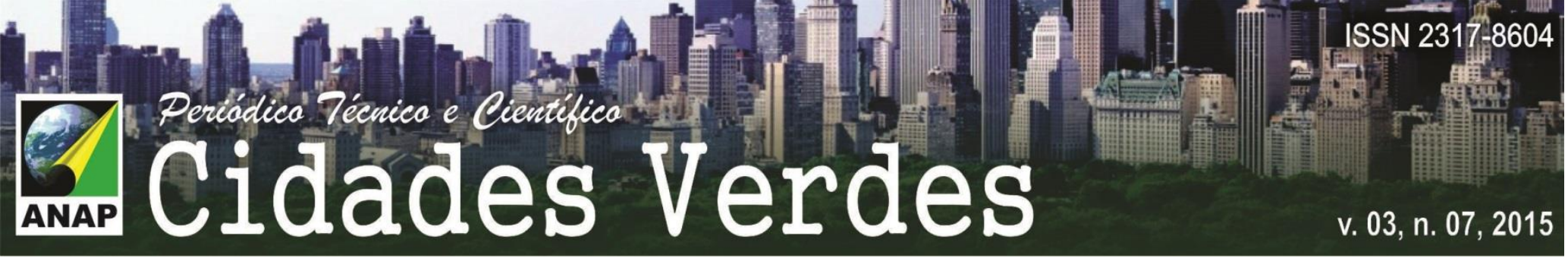

Para o desenvolvimento da mesma, foram realizados levantamentos bibliográficos visando à aquisição de informações relevantes à temática tratada. Assim, foram realizadas leituras em obras, jornais, revistas, trabalhos acadêmicos e, a partir das informações levantadas, foram elaborados resumos e fichamentos com o objetivo de se construir uma base teórica sobre a qual se encaminharia o trabalho. A pesquisa partiu das seguintes etapas:

1aㅡ etapa: Definir a sustentabilidade e o desenvolvimento sustentável, a partir de autores que trabalham com a temática; $2^{\underline{a}}$ etapa: Caracterizar a cidade e destacar os principais acordos e conferências que discutem sobre a sustentabilidade; $3^{\text {a }}$ etapa: Definir a cidade sustentável, e os critérios para análise da mesma, destacando o uso dos indicadores de sustentabilidade; 4⿳亠丷⿵冂丶 etapa: Analisar alguns sistemas de indicadores de sustentabilidade, e propor as ferramentas disponibilizadas pelo Programa Cidades Sustentáveis para auxiliar a gestão pública e/ou estudos acadêmicos na busca de estratégias para o ambiente urbano.

\section{INSERÇÃO DO REFERENCIAL DA SUSTENTABILIDADE NO ESPAÇO URBANO}

\subsection{Sustentabilidade e Cidade - Acordos e Conferências}

Existe uma multiplicidade de definições que se encontra em documentos gerando controvérsias e incertezas sobre o que realmente significa desenvolvimento sustentável e sustentabilidade. De acordo (BARBIERI, 1997) ser sustentável é perceber as soluções para os problemas globais, que não se reduzem apenas à degradação do ambiente físico e biológico, mas que incorporam dimensões sociais, políticas e culturais, como a pobreza e a exclusão social.

A definição mais conhecida e utilizada de desenvolvimento sustentável é a do relatório de Brundtland, sendo:

[...] um processo de transformação, no qual a exploração dos recursos, a direção dos investimentos, a orientação da evolução tecnológica e a mudança institucional se harmonizam e reforçam o potencial presente e 
gerações futuras; participação da população envolvida; preservação dos recursos naturais e do meio ambiente; elaboração de um sistema social que garantisse emprego, segurança social e respeito a outras culturas; programas de educação. Esta teoria referia-se principalmente às regiões subdesenvolvidas, envolvendo uma critica a sociedade industrial. Foram os debates em torno do ecodesenvolvimento que abriram espaço ao conceito de desenvolvimento sustentável (ROSSETO, 2003).

No entanto esse conceito foi definido num processo que antecedeu a Rio 92, sendo amplamente divulgado a partir de 1987 pela Comissão Mundial sobre o Meio Ambiente (CMMAD) - a Comissão Brundtland, como sendo aquele que valoriza o crescimento econômico, mas com a promoção da equidade social e a preservação do patrimônio natural (ALMEIDA, 2011). O Relatório de Brundtland considerou o desenvolvimento sustentável como aquele que atende às necessidades do presente sem comprometer a capacidade das gerações futuras de atender às suas próprias necessidades.

O desafio do desenvolvimento sustentável tornou-se o foco das discussões na Conferência ECO 92 e também teve-se a consolidação da Agenda 21, que preconizou um novo padrão de desenvolvimento para o século $X X I$, baseado na sustentabilidade ambiental, social e econômica (GUILHON, 2011). A Agenda 21 é uma cartilha consensual acordada pela comunidade internacional que assinala alguns programas que farão parte de um plano de ação conjunta para frear a degradação ambiental, quais sejam: atmosfera, recursos da terra, agricultura sustentável, desertificação, florestas, biotecnologia, resíduos sólidos, resíduos tóxicos, entre outros.

Para Guilhon (2011), na Europa, um dos resultados da Agenda 21 foi a realização do I Congresso de Cidades Sustentáveis, em 1994, onde foi acordado que para a aplicação de princípios de desenvolvimento sustentável era necessário o intercambio de experiências, o trabalho em rede e a necessidade de estabelecer indicadores sustentáveis urbanos. 
No entanto, o Programa Cidades Sustentáveis, realizado pela Rede Nossa São Paulo, Rede Social Brasileira por Cidades Justas e Sustentáveis e o Instituto Ethos de Empresas e Responsabilidade Social publicou em 2012 o documento Metas de Sustentabilidade para municípios Brasileiros (Indicadores e Referências), onde apresenta exemplos de boas práticas em diversos municípios que podem servir como referências de metas para o planejamento e a gestão das administrações municipais.

O Programa Cidades Sustentáveis tem como objetivo contribuir com as gestões municipais no sentido da implementação de instrumentos de planejamento e execução de políticas públicas que considerem a sustentabilidade em projetos e ações dos poderes executivos e legislativos municipais, além do devido comprometimento dos setores privados e das sociedades locais.

\section{RESULTADOS E DISCUSSÕES}

A sustentabilidade requer critérios de planejamento e gestão de forma a gerar espaços urbanos justos, democráticos, equitativos, ecológicos, de fácil mobilidade, que garantam serviços de saúde básicos, que preservem os recursos naturais, dentre outros.

Diante dos problemas urbanos que as cidades vem enfrentando, há vários estudos que abordam conceitos relacionados ao desenvolvimento sustentável. Assim, são várias as iniciativas por parte de pesquisadores e organizações para analisar ou mensurar a sustentabilidade de determinados locais através do uso de indicadores. Utilizar indicadores de sustentabilidade são importantes para a gestão pública, pois se tornam uma ferramenta fundamental no processo de avaliação da sustentabilidade.

Para Martins e Cândido (2008), os sistemas de indicadores foram desenvolvidos com o propósito de melhor compreender os fenômenos relacionados à sustentabilidade, sendo poucos os sistemas que atuam com o desenvolvimento sustentável, em sua maioria de caráter experimental. Como afirma Almeida (2011), a complexidade que envolve as questões relacionadas ao desenvolvimento 


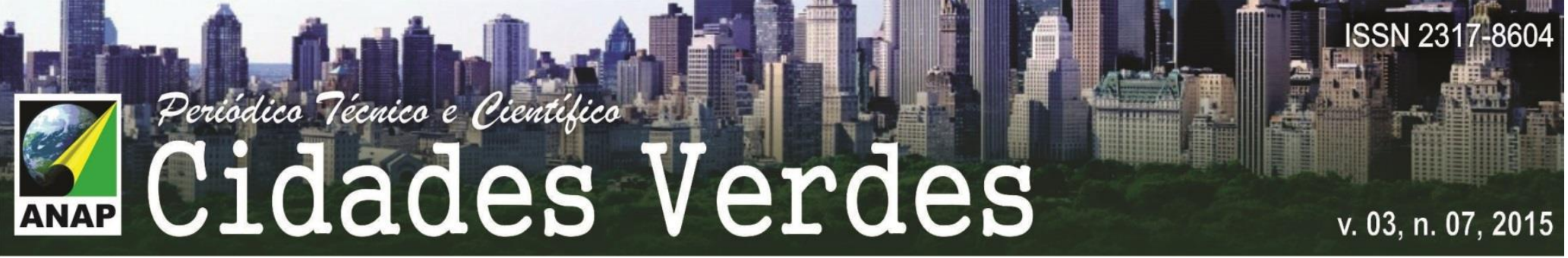

O quadro a seguir, contempla os indicadores básicos desses 12 eixos

temáticos.

Quadro 01: Indicadores e eixos temáticos, *Indicadores não obrigatórios para cidades com menos de 50 mil habitantes, Programa Cidades Sustentáveis, 2012.

\begin{tabular}{|c|}
\hline $\begin{array}{l}\text { GOVERNANÇA } \\
\text { adores: Mulheres empregadas no governo do município, Negros empregados no } \\
\text { rno do município, Pessoas com deficiência empregadas no governo do município, } \\
\text { elhos Municipais, Orçamento executado decidido de forma participativa e Espaços de } \\
\text { sipação deliberativos e audiências públicas na cidade. }\end{array}$ \\
\hline $\begin{array}{l}\text { BENS NATURAIS COMUNS } \\
\text { Indicadores: Área verde por habitante }{ }^{*} \text {, Concentrações de PM10 (material part } \\
\text { MP)*, Concentrações de PM2,5 (material particulado - MP) }{ }^{*} \text {, Concentrações de O3 } \\
\text { Concentrações de CO (monóxido de carbono) }{ }^{*} \text {, Concentrações de NO2 (di } \\
\text { nitrogênio) }{ }^{*} \text {, Concentrações de SO2 (dióxido de enxofre) }{ }^{*} \text {, Perda de água } \\
\text { Abastecimento público de água potável na área urbana, Rede de esgoto, Esgoto }\end{array}$ \\
\hline $\begin{array}{l}\text { ndição } \\
\text { centes, } \\
\text { micídio } \\
\text { ão em }\end{array}$ \\
\hline erentes \\
\hline $\begin{array}{l}\text { Indicadores: Área desma } \\
\text { novos e reformados qu } \\
\text { consideradas adequadas à }\end{array}$ \\
\hline \\
\hline $\begin{array}{l}\text { icadores: Ín } \\
\text { érie, rede } \mathrm{m} \\
\text { Ifabetismo } \mathrm{n}\end{array}$ \\
\hline \\
\hline \\
\hline
\end{tabular}




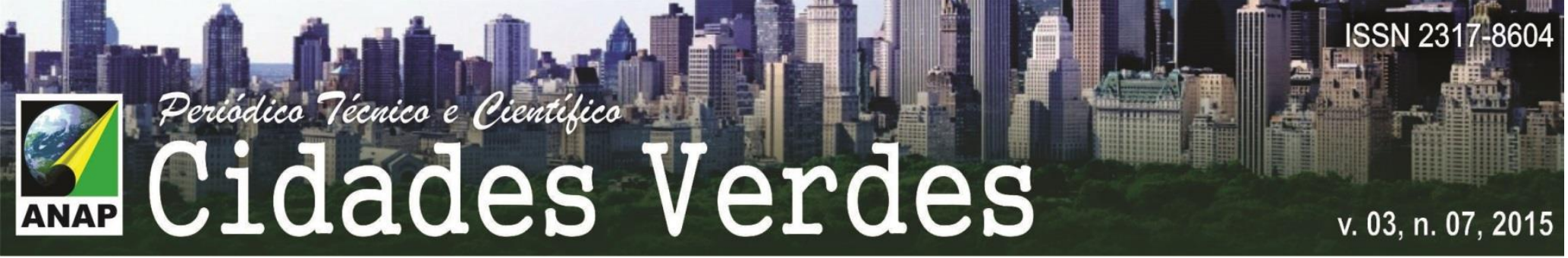

\section{MELHOR MOBILIDADE, MENOS TRÁFEGO}

Indicadores: Corredores exclusivos de ônibus*, Ciclovias exclusivas, Divisão modal, Índice de Congestionamentos* ${ }^{*}$ Frota de ônibus com acessibilidade para pessoas com deficiência, Número de mortes em acidentes de trânsito por 10 mil habitantes, Mortes com bicicleta, Mortes por atropelamento, Mortes com motocicleta, Mortes com automóvel, Acidentes de trânsito, Atropelamento e Orçamento do município destinado a transporte público.

\section{AÇÃO LOCAL PARA A SAÚDE}

Indicadores: Água - Doenças de veiculação hídrica, Pessoas infectadas com dengue, Unidades Básicas de Saúde, Leitos hospitalares, Mortalidade por doenças do aparelho respiratório, Mortalidade por doenças do aparelho circulatório, Gravidez na adolescência, Mortalidade materna, Baixo peso ao nascer, Pré-natal insuficiente, Desnutrição infantil, Mortalidade infantil e Equipamentos esportivos.

\section{DO LOCAL PARA O GLOBAL}

Indicadores: Total de emissões de CO2 equivalente per capita*, Variáveis meteorológicas Temperatura media mensal e Número de Mortes por desastres socioambientais.

Fonte: Programa Cidades Sustentáveis, 2012. Org.: LOPES, A. F. A., 2015.

Os indicadores propostos pelo Programa Cidades Sustentáveis servem como subsídios para as políticas públicas, pois permitem uma compreensão e análise da localidade, através de um conjunto de variáveis ${ }^{4}$ que envolvem a dimensão social, econômica, política, ambiental e cultural. O Método de cálculo é simples e é especificado na Plataforma, a mesma fornece também uma tabela, onde é constituída por eixo, indicadores, descrição, variáveis para compor o indicador e o método de cálculo. Para a compreensão do método de cálculo (Quadro 2), tem-se como exemplo, no eixo Bens Naturais Comuns,o indicador área verde por habitante.

Quadro 02: Eixo, Indicadores, Descrição, Variáveis e Cálculo, Área verde por habitante.

\begin{tabular}{|c|c|c|c|c|}
\hline EIXO & INDICADORES & DESCRIÇÃO & $\begin{array}{lr}\text { VARIÁVEIS PARA } \\
\text { COMPOR } & 0 \\
\text { INDICADOR } & \end{array}$ & $\begin{array}{l}\text { MÉTODO } \\
\text { CÁLCULO }\end{array}$ \\
\hline 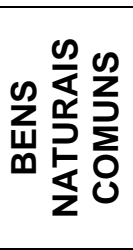 & $\begin{array}{l}\text { Área verde por } \\
\text { habitante }\end{array}$ & $\begin{array}{c}\text { Total de metros } \\
\text { quadrados de área } \\
\text { verde por habitante } \\
\text { (medida anual) Áreas } \\
\text { verdes públicas }\end{array}$ & $\begin{array}{l}\text { Número Total, em } \\
\text { m², de áreas } \\
\text { verdes no } \\
\text { município/ } \\
\text { População Total } \\
\text { do município. }\end{array}$ & $\begin{array}{c}\text { Número Total, em } \\
\mathrm{m}^{2} \text { de áreas verdes } \\
\text { no município \% } \\
\text { População Total do } \\
\text { município. }\end{array}$ \\
\hline
\end{tabular}

Fonte: Programa Cidades Sustentáveis, 2012. Org.: LOPES, A. F. A., 2014.

\footnotetext{
${ }^{4}$ As variáveis dos indicadores e o método de cálculo para avaliar a sustentabilidade municipal são apresentadas no documento Metas de Sustentabilidade para Municípios Brasileiros (Indicadores e Referências) desenvolvido pelo Programa Cidades Sustentáveis.
} 
A plataforma do programa Cidades Sustentáveis permite mensurar variáveis de grande representatividade para um município e também traçar diagnósticos acerca das reais circunstâncias nas quais as variáveis se apresentam.

\section{CONSIDERAÇÕES FINAIS}

Embora as cidades apresentem desenvolvimento econômico e abriguem serviços públicos, as mesmas não deixam de enfrentar problemas urbanos relacionados ao meio ambiente, saúde, educação, mobilidade, moradia, exclusão social, insegurança, violência e outros. Em concordância com Leite (2012), isso ocorre já que, na maioria das vezes, as cidades se desenvolvem baseadas em um modelo insustentável e de uso ineficiente de recursos.

Diante desses problemas urbanos, surgiu o conceito de desenvolvimento sustentável, o mesmo provém de um longo processo histórico de reavaliação da relação existente entre a sociedade civil e o meio natural. Por se tratar de um processo contínuo e complexo, observa-se hoje que existe uma variedade de abordagens que procura explicar o conceito de sustentabilidade, esse fato constatase pela grande quantidade de definições. Por isso, o conceito gera muitas controvérsias e há desacordos entre estudiosos de diferentes disciplinas a respeito de como a sustentabilidade pode ser analisada ou medida.

No documento publicado pelo Programa Cidades Sustentáveis, ressalta-se pelos próprios exemplos bem-sucedidos selecionados, a importância do planejamento técnico aliado aos processos participativos, em que o conhecimento acumulado sobre gestão pública é socializado e posto à prova diante das necessidades e prioridades apontadas pela população local. Por meio do processo participativo, pode-se criar políticas públicas e com responsabilidades compartilhadas entre poderes públicos, sociedade civil e setor privado (PROGRAMA CIDADES SUSTENTÁVEIS, 2012).

Deve-se ressaltar também que um aspecto fundamental ao utilizar os indicadores de sustentabilidade é evitar que os mesmos se tornem instrumentos limitados, refletindo apenas aspectos parciais ou recortes de uma realidade. É 


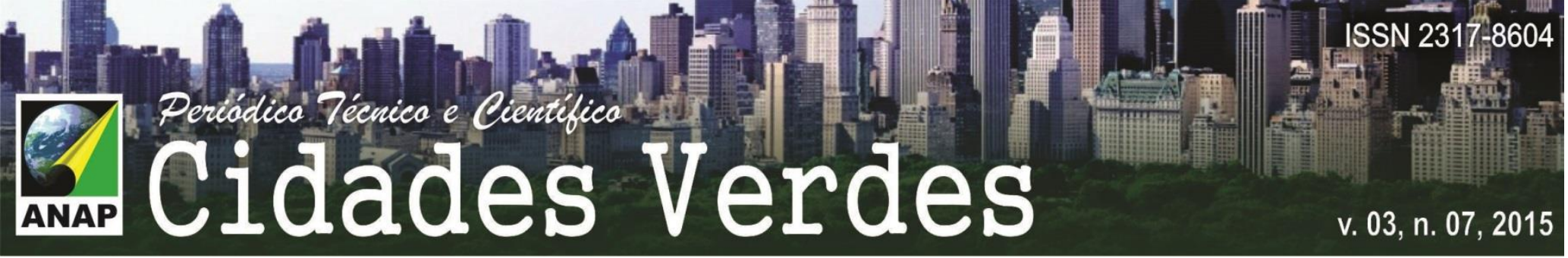

necessário identificar as interligações entre os diversos aspectos relacionados ao conceito de desenvolvimento sustentável e às especificidades locais.

Sabe-se que são grandes os desafios para a sustentabilidade urbana, no entanto, é fundamental o envolvimento dos cidadãos, organizações sociais, empresas e governos. Espera-se que esse trabalho contribua para a gestão pública municipal, pois com o uso de indicadores no processo de avaliação do ambiente urbano, os mesmos fornecem informações que podem auxiliar nas políticas de desenvolvimento sustentável. Espera-se também que o presente trabalho contribua para futuras pesquisas acadêmicas que visem analisar a sustentabilidade em municípios, cidades ou determinadas localidades, através das ferramentas que são propostas pelo Programa Cidades Sustentáveis.

\section{REFERÊNCIAS}

ACSELRAD, H. Sentidos da Sustentabilidade Urbana. Salvador, BA, 2005.

AGUIAR, I. G. Cidades Sustentáveis: Desafios e Propostas. Fragmentos de Cultura, V.18. Goiânia, 2008.

ALMEIDA, A. C. Índice de desenvolvimento sustentável municipal participativo: um estudo da sustentabilidade do município de Pitimbu a partir da percepção de atores sociais. $156 \mathrm{f}$. Dissertação (Mestrado em Recursos Naturais)- Universidade Federal de Campina Grande, Campina Grande, 2011.

BARBIERI, J. C. Desenvolvimento e Meio Ambiente: as estratégias de mudança da agenda 21. Petrópolis: Vozes, 1997.

BRANDÃO, R. S. As interações espaciais urbanas e o clima. $351 \mathrm{f}$. Tese (Doutorado em Arquitetura e Urbanismo)- Universidade Federal de São Paulo, São Paulo, 2009.

CMMAD - Comissão Mundial sobre Meio Ambiente e Desenvolvimento. Nosso futuro comum. 2a ed. Tradução de Our common future. 1a ed. Rio de Janeiro : Editora FGV, 1988.

CORRÊA, R. L. Processos Espaciais e a Cidade. In: Trajetórias Geográficas. Rio de Janeiro: Bertrand Brasil, 1997, p. 121-143.

GIBBERD, J. Building systems to support sustainable development in developing countries. CSIR, Division of Building and Construction Technology Pretoria, 2003.

GIL, A. C. Como Elaborar Projetos de Pesquisa. 4. Ed. São Paulo: Atlas, 2002.

GUILHON, V. V. Indicadores de Sustentabilidade Urbana: Aplicação ao conjunto habitacional ‘ Parque Residencial Manaus/ AM'. 217 f. Dissertação (mestrado em Arquitetura e Urbanismo)Faculdade de Arquitetura e Urbanismo da Universidade de São Paulo, São Paulo, 2011.

IBGE- Instituto Brasileiro de Geografia e Estatística. Indicadores de desenvolvimento sustentável: 


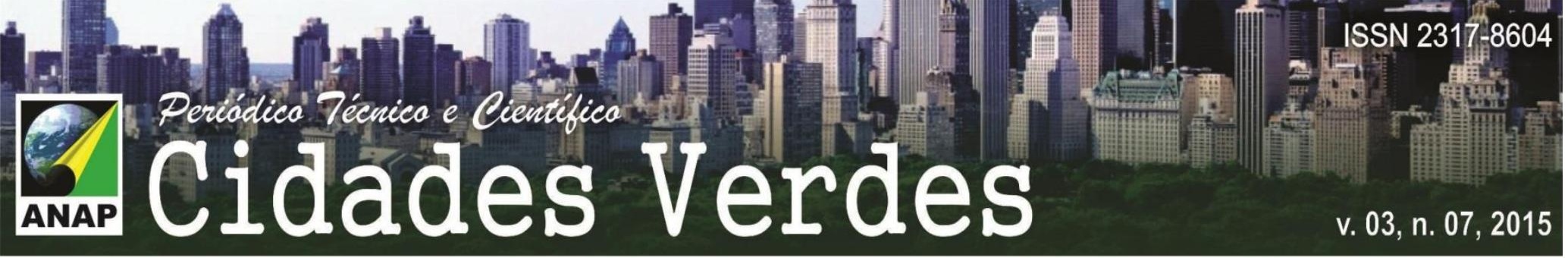

Brasil 2010 (IDS 2010). Rio de Janeiro, 2010.

LEITE, C. Cidades Sustentáveis, Cidades Inteligentes: Desenvolvimento Sustentável em um Planeta Urbano. Porto Alegre: Bookman, 2012.

MINAYO, Maria Cecília de Souza et al (org.). Pesquisa social: teoria, método e criatividade. 19å. ed. Rio de Janeiro: Vozes, 2006.

MUMFORD, L. A cidade na história: suas origens, transformações e perspectivas. São Paulo: Marins Fontes Editora, 1991.

PROGRAMA CIDADES SUSTENTÁVEIS. Indicadores. São Paulo, 2012. Disponível em: <http://www.cidadessustentaveis.org.br/>. Acesso em: 14 jan. 2015.

ROGERS, Richard; GUMUCHDJIAN, Philip. Cidades para um pequeno planeta. Barcelona: GG, 2008.

ROSSETO, A. M. Proposta de um Sistema Integrado de Gestão do Ambiente Urbano (SIGAU) para o desenvolvimento sustentável de Cidades. $404 \mathrm{f}$. Tese (Doutorado em Engenharia de Produção)-. Universidade Federal de Santa Catarina, Florianópolis, 2003.

VAN BELLEN, H. M. Indicadores de Sustentabilidade: uma análise comparativa. Editora FGV. Rio de Janeiro, 2005. 